\title{
Precious, Tiny and Shiny: Close-range Photogrammetry for Artists and Others
}

\author{
Naomi Dines \\ Fine Art Programme, Central St Martins \\ London, UK \\ n.dines@csm.arts.ac.uk
}

\section{INTRODUCTION}

Photogrammetry - deriving '3D models' with photorealistic surface 'textures' from digital images - is increasingly being used to capture digital assets for creative projects, but still presents challenges for certain types of object. This short paper outlines the ongoing development of apparatus, methods and devices that offer affordable, reliable, and scalable solutions for high-resolution digital capture with a single DSLR camera. These approaches are helping to open up creative possibilities further down the virtual or material 'pipeline' by creating accurate 'digital objects' for a variety of uses from virtual reality (VR) to computer-aided manufacture (CAM).

\section{CAPTURE, CREATION, PRODUCTION}

The artistic element of this project seeks to capture, re-examine and ultimately transform a range of military and religious insignia; cultural artefacts that share specific materials and processes, as well as powerfully interrelated iconographies and histories. These objects bear the traces of their making, use and age in minute material detail. Their reflective metal embroidery forms complex image-objects, often on richly textured fabric grounds, presenting a particular challenge for their digital capture.

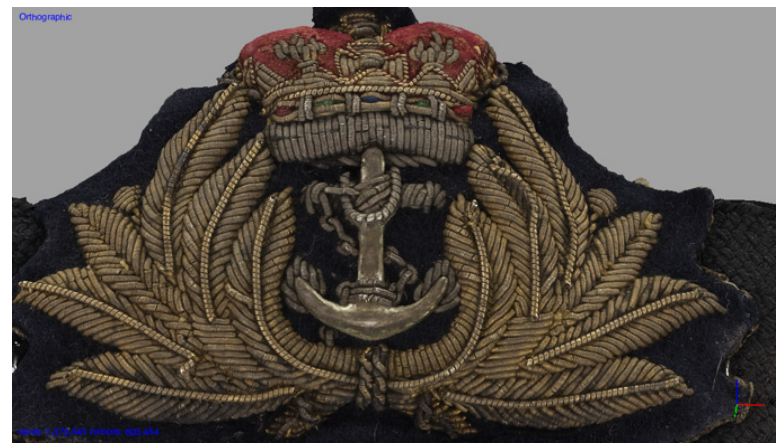

Figure 1: Textured Model of 1950s Naval cap badge

\subsection{Methods, devices and solutions}

Such small and highly detailed objects lend themselves to the more technically demanding 'rotating object / static camera' approach. Carefullyaligned, orthographic camera positions and controlled angular rotation of objects significantly increased the accuracy and speed of captures and also allowed rapid visual analysis of the resulting image alignment during processing. Using Agisoft's 'Photoscan Pro' software, misaligned images could be easily identified in the first stages of processing and successfully realigned, aiding accurate model reconstruction.
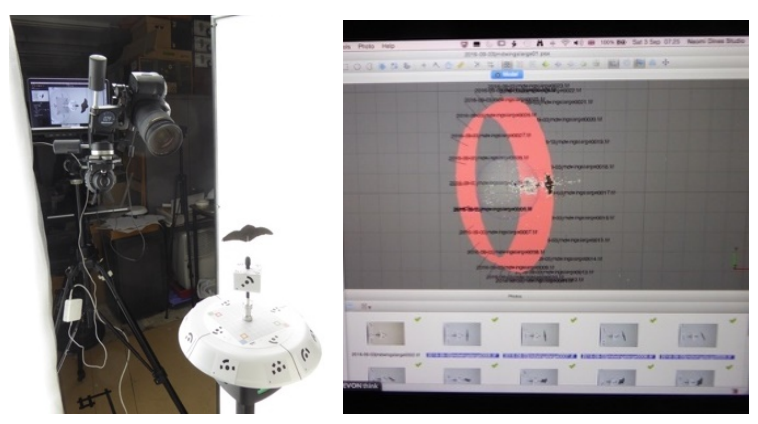

Figure 2: Early ortho-alignment and clean image ring

Very diffuse and low-intensity lighting allowed the capture of often highly reflective objects without the need for expensive polarising filters for lights and lenses. Good 3D captures also require excellent focus across source images. Prime macro lenses and focus stacking were used to improve depth of field in low light, enhancing the photogrammetric 'alignment' of image sets and the resulting model accuracy, surface quality and texture resolution. Highly specific depth of field also allowed effectively featureless backgrounds to be created in-camera, helping the algorithms to triangulate $3 \mathrm{D}$ structure from the 'isolated' relative motion of camera and object in successive images. Careful 
control of background, lighting and focal depth also enabled rapid masking of images in photogrammetry software, either automatically through a pre-captured or subsequently created 'empty' background image, or through the programme's own masking tools.

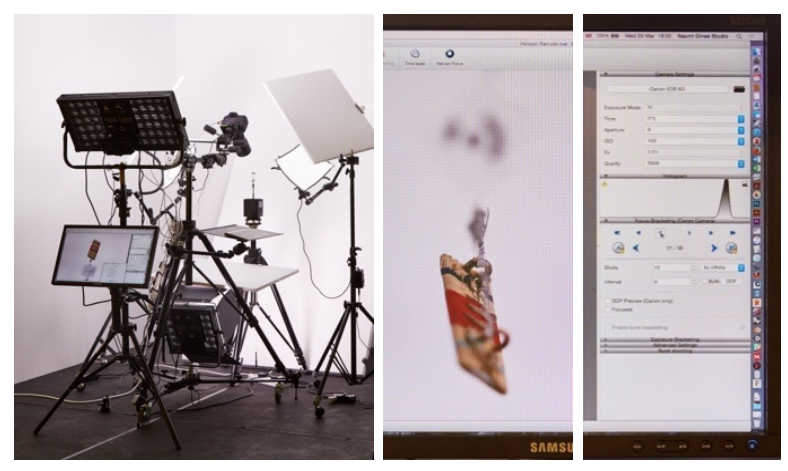

Figure 3: Tethered focus stacking with Helicon Remote

Remote camera operation and tethered shooting made setting up shoots, capturing and organising image data-sets more controllable, precise and efficient. 'Helicon Remote' software allowed automated focus-bracketing of several images for each camera / object position, and these were then batch-processed into single 'focus stacked' images using 'Helicon Focus' before being aligned in 'Agisoft's dedicated photogrammetry software 'Photoscan Pro'. With these approaches it was possible to achieve extremely accurate, relatively high-polygon models with no mesh 'noise', and very high resolution photo-realistic surface textures.

\subsection{Increasing accuracy and efficiency}

Geared tripod columns, and carefully designed assemblies of other commonly available photographic equipment, have allowed object / camera loci that reduce the range of relative motion between camera and object and the consequent alignment required, greatly increasing the speed and reliability of captures. Following initial experiments with more basic manual and powered turntables, these were replaced with readily available photographic 'motion controllers', able to be mathematically synced with the focus stacking software to allow 'one-click' captures for each camera angle.

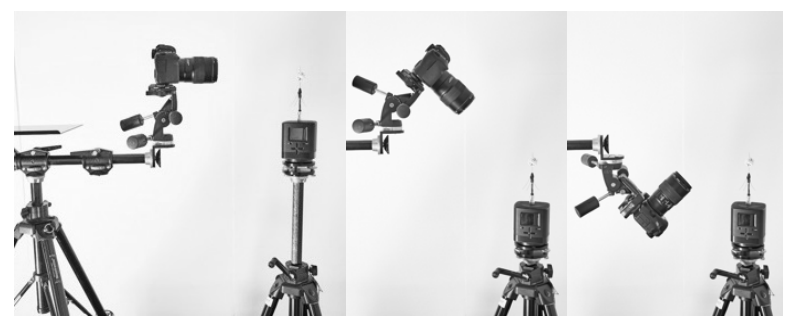

Figure 4: Geared-columns: camera/object relative motion
Coded and non-coded targets are used extensively in archaeology, anthropology and paleontology to aid the alignment of images and reconstruction of fragmentary captures into whole objects or unified terrains. By developing prototype 3-dimensional computer-coded devices with appropriate camerasensor-to-target scales and face angles, it has been possible to use automated target recognition to help reliably capture small, symmetrical, monochrome or reflective artifacts that cannot be treated with surface sprays, or marked to assist with feature recognition.

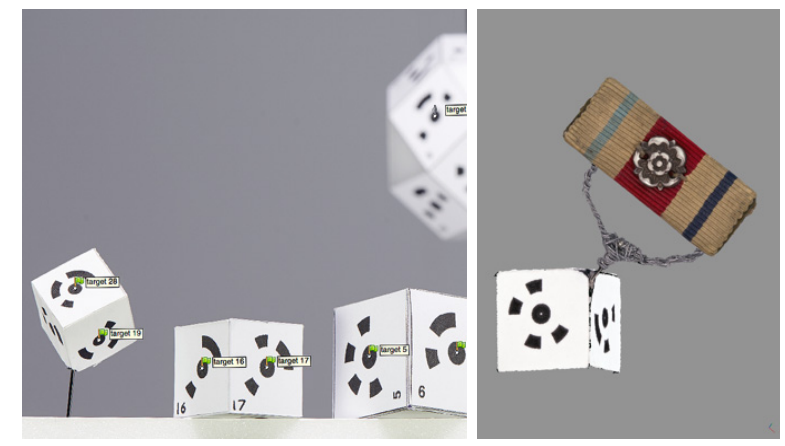

Figure 5: Coded target objects \& WWII Medal Bar model

\subsection{New digital and material objects}

By overcoming the limitations of standard capture techniques it has been possible to create highly accurate and detailed 'digital objects', equivalent to the hyper-real resolutions more common in staticand moving digital imagery in photography and cinema, as well as in art works. Almost infinite zooming and scaling of these highly detailed models make it possible to use gaming techniques to interact with and navigate around 'virtual' objects without the loss of an 'life-like' level of photorealism, opening up the potential to create new audience experiences of specific objects and their conceptual and creative derivatives. These capture methods will also support the production of physical objects only made possible with 3D modelling and advanced digital manufacture, and their ability to extend and hybridise more traditional techniques.

\section{REFERENCES (SELECTED)}

Keenan, T. and Weizman, E. (2012) Mengele's Skull: The Advent of a Forensic Aesthetics. Sternberg Press/Portikus, Berlin/Frankfurt am Main.

Mallison, H. and Wings, O. (2014) Photogrammetry in paleontology - a practical guide. Journal of Paleontological Techniques, 12, pp. 1-31.

Wijenayake, U. et al (2014) Automatic detection and decoding of photogrammetric coded targets. https://arxiv.org/abs/1601.00396 (14 May 2016). 